Revista Colombiana de Obstetricia y Ginecología Vol. 61 No. 1 • $2010 \bullet(66-71)$

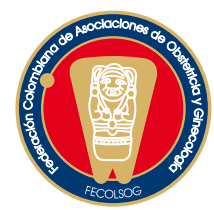

Reporte de caso

EMBARAZO ECTÓPICO EN LA CICATRIZ UTERINA: REPORTE DE UN CASO Y REVISIÓN DE LA LITERATURA

\title{
Ectopic pregnancy in the uterine scar: a case report and literature review
}

Janer Sepúlveda-Agudelo, M.D. *, Mayra Cristancho-Solano, M.D.**, Carolina Parra-Meza, M.D.***

Recibido: julio 30/09 - Aceptado: febrero 15/10

\section{RESUMEN}

Introducción: el embarazo ectópico en la cicatriz uterina se presenta en 1:1.800 a 1:2.216 embarazos y representa $6,1 \%$ de los embarazos ectópicos en pacientes con antecedente de cesárea.

Objetivo: revisar el manejo y el diagnóstico del embarazo ectópico en la cicatriz uterina a partir del caso de una paciente con esta patología.

Presentación del caso: una paciente de 24 años con antecedente de cesárea, asistió a consulta en el Hospital Universitario de Santander con dolor en el hemiabdomen inferior. A partir de estos síntomas, se le realizó ecografía y Doppler interpretados como de alta probabilidad de embarazo ectópico en la cicatriz uterina. Posteriormente, se inició terapia con metotrexato combinado con ácido folínico. Finalmente, se hizo control con evolución favorable de la paciente.

Discusión: el embarazo ectópico en la cicatriz uterina es una complicación a largo plazo de la cesárea. Su diagnóstico debe ser considerado en toda mujer con

* Médico Gineco-obstetra. Profesor Asistente, Escuela de Medicina, Universidad Industrial de Santander. Miembros del Grupo de Investigación GINO. Bucaramanga (Colombia). Correo electrónico: janersepulveda@yahoo.es

** Médico Gineco-obstetra. Universidad Industrial de Santander. Bucaramanga, (Colombia).

*** Médico Gineco-obstetra. Universidad Industrial de Santander Bucaramanga (Colombia). antecedente de cesárea que consulte por hemorragia en la primera mitad del embarazo antes de la décima semana de gestación. En estos casos, el diagnóstico ecográfico inicial puede ser difícil pero se puede llegar a un diagnóstico certero con ecografías repetidas. Por otra parte, el tratamiento puede ser médico o quirúrgico y depende básicamente del tiempo de gestación en que se haga el diagnóstico.

Palabras clave: embarazo ectópico, embarazo en la cicatriz de cesárea, parto por cesárea.

\section{SUMMARY}

Introduction: ectopic pregnancy in the uterine scar occurs in 1:1,800 to $1: 2,216$ of pregnancies and accounts for $6.1 \%$ of ectopic pregnancies in the group of patients having had prior cesarean section.

Objective: in order to review the management and diagnosis of this condition, the case of a patient having an ectopic pregnancy in the uterine scar is presented.

Case presentation: a 24-year-old patient having had a previous cesarean section consulted the Santander teaching hospital suffering from lower abdominal pain. Doppler ultrasound suggested a high probability of ectopic pregnancy in the uterine scar. Methotrexate and folinic acid therapy was thus begun. Controls were made with favorable results. 
Discussion: ectopic pregnancy in the uterine scar is a long-term complication of cesarean section; it is a diagnosis which should be considered in any female having a history of previous cesarean consulting for hemorrhage during the first half of pregnancy before 10 weeks' gestation. Initial ultrasound diagnosis can be difficult; however, diagnosis can be made with repeated scans. Treatment may be medical or surgical and will mainly depend on the gestation time when diagnosis is made.

Key words: ectopic pregnancy, cesarean scar pregnancy, cesarean delivery.

\section{INTRODUCCIÓN}

El embarazo ectópico se caracteriza por la implantación del blastocisto fuera de la cavidad uterina. Esta condición se presenta entre 1\% y 2\% de todos los embarazos y es la principal causa de muerte obstétrica en el primer trimestre de gestación. ${ }^{1}$ Se estima que 15 de cada 1.000 embarazos en Colombia son ectópicos y 90\% de éstos se localizan en la trompa de falopio mientras que $10 \%$ de los casos restantes se presentan en otras partes como el ovario, el abdomen, la cerviz y la cicatriz uterina. ${ }^{2}$

En contraste, el embarazo ectópico en la cicatriz uterina ocurre con la implantación del blastocisto en el área de la cicatriz del útero, rodeado por el miometrio y el tejido conectivo. ${ }^{3}$ Éste se presenta en 1:1.800 a 1:2.216 embarazos y representa 6,1\% de los embarazos ectópicos en pacientes con antecedente de cesárea. ${ }^{3}$ Dicha incidencia es mayor que la del embarazo ectópico cervical que ocurre en 1:2.000 a 1:18.000 embarazos. ${ }^{3}$ Debido a la baja incidencia de este evento, la información disponible acerca del diagnóstico, tratamiento y pronóstico deriva de estudios observacionales con muestras pequeñas y reportes de casos. ${ }^{4}$

Por consiguiente, el objetivo de la presentación de este caso, manejado en el servicio de ginecología y obstetricia del Hospital Universitario de Santander en Bucaramanga (Colombia), es hacer una revisión sobre el embarazo ectópico en la cicatriz uterina con énfasis en su diagnóstico y tratamiento.

\section{PRESENTACIÓN DEL CASO}

La paciente de 24 años, una ama de casa con antecedente de una gestación y una cesárea, ingresó el día 4 de diciembre de 2008 al Hospital Universitario de Santander (centro de referencia regional en la zona noroccidental del país). Se llevó a cabo consulta por dolor tipo cólico en el hemiabdomen inferior asociado con sangrado vaginal y de 5 días de evolución. La paciente también presentaba 10 semanas de amenorrea.

En el examen físico de ingreso la paciente, alerta y estable hemodinámicamente, presentó útero grávido aumentado como para 8 semanas con cérvix cerrado, posterior, abombado y lateralizado a la derecha. El resto del examen se encontró dentro de los límites normales. Asimismo, se consideró un cuadro compatible con amenaza de aborto, por lo cual se practicó un ultrasonido pélvico por vía transvaginal, indicando un útero de tamaño aumentado con eco endometrial de $11 \mathrm{~mm}$ bilaminar. En el istmo, el cual tenía un grosor de $57 \mathrm{~mm}$, se observaron ecos endometriales mixtos de $67 \mathrm{~mm}$ x $50 \mathrm{~mm}$ x $64 \mathrm{~mm}$ en forma de reloj de arena. Se realizó diagnóstico de aborto incompleto, sin embargo, no se descartó la posibilidad de embarazo ectópico cervical o de la cicatriz uterina.

Finalmente, se realizaron varios exámenes paraclínicos, entre ellos una prueba de fracción $\beta$ cuantitativa de la Hormona Gonadotrofina Coriónica Humana (B-HCG) que arrojó los siguientes datos: título de 24,188 $\mathrm{mUI} / \mathrm{mL}$; hemoglobina de $12,3 \mathrm{~g} / \mathrm{dL}$, leucocitos de $5.600 \times \mathrm{mm}^{3}$ (segmentados: 79\%, linfocitos: $18 \%$ ) y plaquetas de $223.000 \mathrm{x} \mathrm{mm}^{3}$.

Por otra parte, la paciente presentaba sangrado persistente por lo que se le realizó legrado obstétrico sin complicaciones. Asimismo, se extrajo 40 cc de material membranoso y se tomó una muestra para patología. Tiempo después, la mujer mostró signos de una buena evolución clínica, por lo cual se autorizó su salida.

Después de 8 días ella regresó para realizarse un control con ecografía que mostró un útero de tama- 
ño aumentado con eco endometrial de $9 \mathrm{~mm}$ monolaminar. En cuanto a la pared anterior del istmo, se observó una imagen heterogénea de 45 mm x 42 mm compatible con un embarazo ectópico de la cicatriz uterina. A raíz de esto, se solicitó una prueba de la B-HCG cuantitativa que evidenció $943 \mathrm{mUI} / \mathrm{mL}$ y un reporte de patología, indicando un endometrio en fase secretora con reacción de Arias Stella y deciduitis aguda moderada.

El 18 de diciembre de 2008 se tomó la decisión de hospitalizar a la paciente debido a la persistencia del sangrado. En primer lugar, se efectuó un cuadro hemático de control que evidenciaba $\mathrm{Hb}$ de 7,6 g/dL por lo que se le realizó una transfusión de 2 unidades de glóbulos rojos empaquetados (UGRE). Luego, se llevó a cabo una ecografía que reveló una imagen hipoecogénica, ocupando cavidad, con bordes bien definidos de $61 \mathrm{~mm}$ x $53 \mathrm{~mm}$ que hacían parte de la región segmentaria del útero. Figura 1. También se llevó a cabo un Doppler en donde se observó imagen con alto flujo, velocidad alta de $30 \mathrm{~cm} / \mathrm{s}$, resistencia baja (alta vascularización) y ausencia de infiltración vesical. Figura 2. A causa de esto, se estimó una probabilidad alta de embarazo ectópico en la cicatriz uterina.

Ahora bien, dado el deseo de fertilidad futura por parte de la paciente, la estabilidad hemodinámica y la ausencia de irritación peritoneal se consideró

Figura 1. Ecografía del embarazo ectópico de la cicatriz uterina.

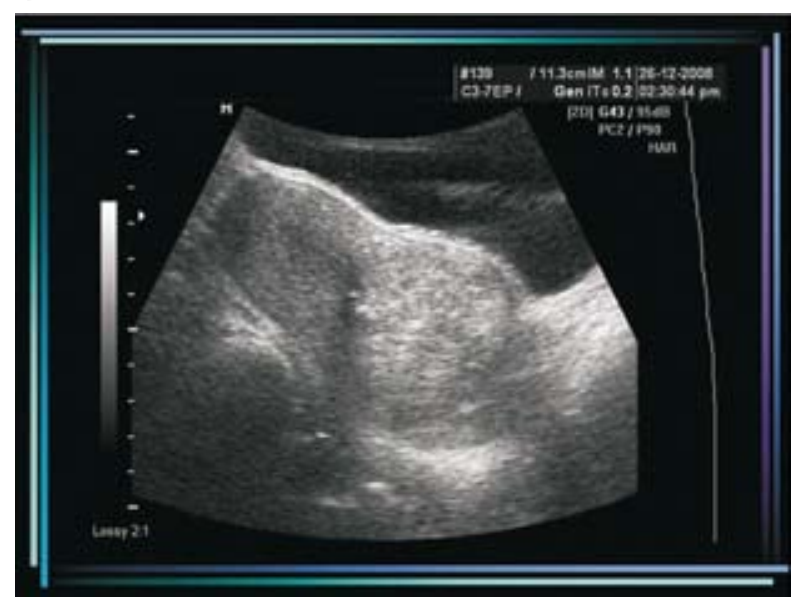

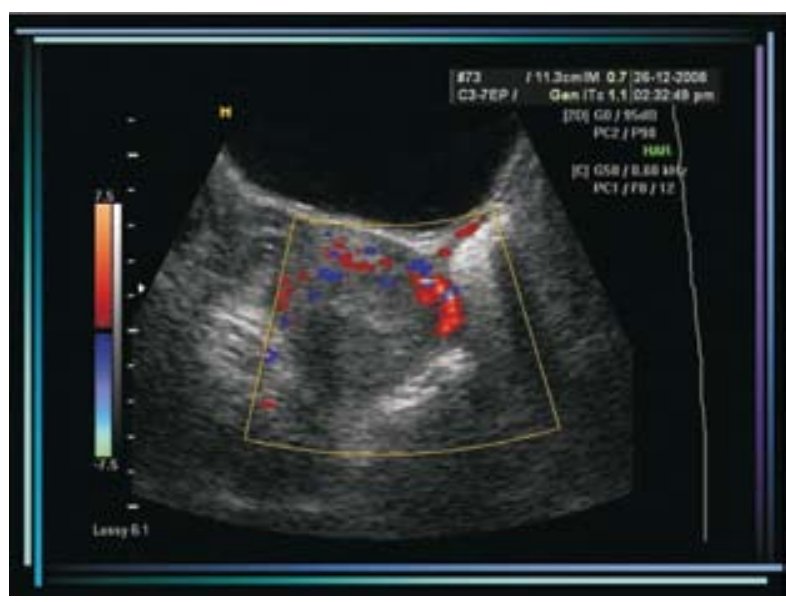

Figura 2. Doppler del embarazo ectópico de la cicatriz uterina.

dar terapia con metotrexate combinado con ácido folínico. Una vez terminado el tratamiento, se llevó a cabo una prueba de la B-HCG cuantitativa de control con un reporte de 199,9 mUI/mL y una ecografía de control que mostró una masa heterogénea de $44 \mathrm{~mm}$ x $41 \mathrm{~mm}$ en la pared anterior a nivel de la cicatriz uterina.

Posteriormente, se dio de alta a la paciente y se le explicó que la resolución completa podía durar hasta 6 semanas. También se le citó por consulta externa 10 días después del tratamiento. En ésta, la mujer refirió persistencia del sangrado, cérvix abombado cerrado y útero de tamaño ligeramente aumentado, por lo que se le practicó nuevamente una prueba de la B-HCG cuyo resultado fue $19 \mathrm{mUI} / \mathrm{mL}$. Igualmente, se efectuó una ecografía en la cual se observó un útero de tamaño normal con eco endometrial de $8 \mathrm{~mm}$ monolaminar e imagen heterogénea a nivel de la cicatriz uterina de $36 \mathrm{~mm}$ x $30 \mathrm{~mm}$, compatible con la resolución del embarazo ectópico en la cicatriz del útero. Después de esto, ella no asistió a los controles programados.

\section{METODOLOGÍA}

Se buscó información de los últimos 10 años en las bases de datos Pub Med/MEDLINE y Ovid con las palabras clave: "ectopic pregnancy" y "cesarean scar pregnancy”. Dado que esta patología se presenta con 
poca frecuencia, se escogieron las publicaciones de texto completo a las que se obtuvo acceso y las cuales hacían énfasis en el diagnóstico y el tratamiento del embarazo ectópico de la cicatriz uterina.

\section{DISCUSIÓN}

El embarazo ectópico localizado en la cicatriz uterina es la forma menos común de embarazo ectópico. Esta patología se reportó por primera vez en 1978 por Larsen y Solomo, y se caracteriza por la implantación del blastocisto en el área de la cicatriz uterina, rodeado completamente del miometrio y del tejido conectivo. $^{5}$ La edad materna promedio es de 33,4 años y la edad gestacional promedio en el momento del diagnóstico es de 7,5 +/- 2,5 semanas. ${ }^{3}$

De igual modo, la fisiopatología no está del todo establecida pero se considera que el mecanismo de implantación está relacionado con la migración del blastocisto a través de fístulas microscópicas presentes en la cicatriz uterina o en la histerotomía. ${ }^{6}$ Estos defectos pueden ser detectados por ultrasonografía aproximadamente dos meses después del procedimiento quirúrgico. Por ejemplo, en un estudio realizado en 47 pacientes a las que se les realizó cesárea, se observó que 50\% de ellas presentaban pequeñas dehiscencias de la cicatriz uterina 3 meses después del procedimiento. ${ }^{3}$

Con respecto a los factores de riesgo para esta condición se incluyen: cicatriz uterina secundaria a operación cesárea o miomectomía, especialmente en pacientes con período intergenésico corto o endometritis postcesárea $;{ }^{7}$ uso de técnicas de fertilización "in vitro", adenomiosis, antecedente de curetaje uterino y revisión uterina. Aunque algunos autores consideran que el número de cesáreas no tiene relación directa con el riesgo de embarazo ectópico de la cicatriz uterina, en una revisión de 112 casos realizada en el 2006 se encontró que 52\% de las pacientes con embarazo ectópico en la cicatriz uterina presentaban antecedente de una cesárea y 72\% habían tenido dos o más cesáreas. ${ }^{8,9}$ Asimismo, entre las manifestaciones clínicas se encuentran diferentes grados de sangrado vaginal asociado con dolor pélvico en relación a la ruptura uterina y al shock hipovolémico secundario. ${ }^{5}$ Sin embargo, este cuadro clínico completo se presenta sólo en 8 de los casos, ya que en la mayoría (40\%) se observa sangrado vaginal con dolor hipogástrico leve o sin dolor pélvico y en $18 \%$ de los casos las pacientes son asintomáticas. ${ }^{6}$

Por otro lado, el diagnóstico se realiza con ultrasonografía pélvica por vía transvaginal con sensibilidad de 84,6\%. ${ }^{3}$ Los hallazgos incluyen visualización de la cicatriz uterina aumentada en tamaño con la presencia de una masa tipo saco gestacional dentro de la misma, localizada en la pared anterior del útero y rodeada de miometrio con un grosor $2 \mathrm{~mm}$ y $5 \mathrm{~mm} .{ }^{10}$ Los criterios diagnósticos ecográficos fueron descritos por Fylstra y Godin $^{5}$ y son especialmente útiles para diferenciar el embarazo ectópico en la cicatriz uterina de un embarazo ístmico cervical: ${ }^{7}$

1) Ausencia de embrión dentro de la cavidad uterina y en el canal cervical.

2) Desarrollo del saco gestacional en la porción anterior baja del segmento uterino.

3) Ausencia de miometrio sano entre el saco gestacional y la vejiga con discontinuidad de la pared uterina anterior en un corte sagital. ${ }^{11}$

Ahora bien, el estudio con ultrasonografía Doppler que evidencia un aumento de la vasculatura peritrofoblástica ${ }^{7,8}$ puede ser útil en la realización de un diagnóstico más certero. ${ }^{7}$ Una vez realizado el diagnóstico se debe descartar el compromiso de estructuras pélvicas adyacentes como la vejiga. Por otra parte, la Resonancia Magnética Nuclear (RMN) y la histeroscopia pueden ser herramientas diagnósticas complementarias, aún así no son indispensables ya que el diagnóstico y la extensión del proceso se pueden realizar a través de una ultrasonografía. ${ }^{7,9}$ El diagnóstico diferencial incluye embarazo ectópico cervical y acretismo placentario.

El tratamiento es individualizado, pues se deben considerar factores como el deseo de fertilidad, el 
tamaño del saco gestacional, la edad gestacional y el estado hemodinámico. ${ }^{12}$ De igual modo, la selección del tratamiento depende de la condición clínica de la paciente, los niveles de B-HCG y la experiencia quirúrgica. También es necesario que a la paciente se le expliquen muy bien las opciones de tratamiento (riesgo de ruptura uterina, hemorragia y posibilidad de histerectomía). ${ }^{13}$ Las opciones de manejo incluyen:

1) Resección quirúrgica del saco gestacional ectópico por laparotomía y reparación de la cicatriz uterina. ${ }^{3,5}$ Recientemente, Wang y sus colaboradores publicaron una serie de 11 casos entre los cuales 4 fueron manejados por videolaparoscopia, 6 por histeroscopia y en un caso se combinaron las 2 técnicas. En ninguno de los casos se reportaron complicaciones quirúrgicas. ${ }^{14}$ Esta opción permite remover el embarazo, reparar el defecto uterino y disminuir el riesgo de hemorragia secundario a ruptura si falla el manejo médico. Debido a que éste último tiene muy baja efectividad y existe un mayor riesgo de complicaciones, el manejo quirúrgico es indicado si el embarazo es mayor de 8 semanas o los niveles de la B-HCG son mayores de $15.000 \mathrm{mU} / \mathrm{mL}^{6,15}$

2) Manejo médico con inyección local de cloruro de potasio o metotrexato dentro del saco gestacional (en especial si existe actividad cardíaca) o metotrexato en forma sistémica con esquema de dosis única o múltiple. ${ }^{3,5,9}$ Esta modalidad de tratamiento se recomienda cuando los niveles de B-HCG son menores de $5.000 \mathrm{mU} / \mathrm{mL}$ o el grosor de la línea miometrial entre la vejiga y el saco gestacional es menor de $2 \mathrm{~mm} \cdot{ }^{8,13}$ No obstante, se han descrito casos exitosos en los que se ha empleado metotrexato local más sistémico en niveles de B-HCG hasta de $46.000 \mathrm{mUI} / \mathrm{mL}^{3}$. Se debe tener en cuenta, asimismo, que al utilizar metotrexato las pacientes no deben presentar afecciones en curso como enfermedad hepática o renal, trombocitopenia o leucopenia. ${ }^{16}$
3) La embolización de las arterias uterinas se ha empleado para reducir el riesgo de hemorragia en pacientes seleccionadas para manejo conservador. $^{3}$

4) A excepción de los casos en los que se demuestre una conexión entre el saco y la cavidad uterina, actualmente no se recomienda la dilatación y el curetaje debido al riesgo de sangrado masivo, la baja efectividad en la resolución del proceso y el riesgo de perforación y ruptura que requiere la histerectomía. ${ }^{3,5,7}$

5) El manejo expectante está contraindicado debido a que el riesgo de ruptura y el sangrado masivo ponen en riesgo la vida de la paciente. ${ }^{3,7}$

De otra parte, el seguimiento se realiza con niveles de B-HCG semanal hasta su negativización y ecografías mensuales que se realizan hasta que no se visualicen productos de la concepción. Cuando se lleva a cabo un manejo médico se considera que la mayoría de los casos presentan resolución total entre 6 y 9 meses, teniendo en cuenta que la absorción del saco gestacional es muy lenta ya que la placenta se implanta en tejido fibroso. Los indicadores de falla en el tratamiento que requieren manejo quirúrgico son la persistencia de niveles elevados de B-HCG, la actividad cardíaca fetal o el crecimiento del saco. ${ }^{3}$

Con respecto al pronóstico, el riesgo de recurrencia es de $30 \%$, ya que se han descrito casos de embarazos a término después de embarazo ectópico de la cicatriz uterina. Pese a esto, el riesgo de ruptura uterina y de acretismo placentario es muy alto. En este caso, las pacientes deben ser llevadas a cesárea al completar las 37 semanas de gestación. ${ }^{5}$

\section{CONCLUSIÓN}

El embarazo ectópico en la cicatriz uterina es una complicación de la cesárea a largo plazo. Su diagnóstico debe considerarse en toda mujer con historia de cesárea previa. Asimismo, el manejo puede ser médico o quirúrgico, dependiendo básicamente del tiempo de gestación en que se haga el diagnóstico. 


\section{REFERENCIAS}

1. Chang J, Elam-Evans LD, Berg CJ, Herndon J, Flowers L, Seed KA, et al. Pregnancy-related mortality surveillance. United States, 1991-1999. MMWR Surveill Summ 2003;52:1-8.

2. Murray H, Baakdah H, Bardell T, Tulandi T. Diagnosis and treatment of ectopic pregnancy. CMAJ 2005;173:905-12.

3. Rotas MA, Haberman S, Levgur M. Cesarean scar ectopic pregnancies, etiology, diagnosis, and management. Obstet Gynecol 2006;107:1373-81.

4. Molinaro TA, Barnhart KT. Abdominal pregnancy, cesarean scar pregnancy and heterotopic pregnancy. UpToDate. May 2008.

5. Fylstra DL. Ectopic pregnancy within a cesarean scar: a review. Obstet Gynecol Surv 2002;57:537-43.

6. Molinaro TA, Barnhart KT. Ectopic pregnancies in unusual locations. Semin Reprod Med 2007;25:123-30.

7. Holland MG, BienstockJL. Recurrent ectopic pregnancy in a cesarean scar. Obstet Gynecol 2008;111:541-5.

8. Maymon R, Halperin R, Mendlovic S, Schneider D, Herman A. Ectopic pregnancies in a cesarean scar: review of the medical approach to an iatrogenic complication. Hum Reprod Update 2004;10:515-23.

9. Ash A, Smith A, Maxwell D. Caesarean scar pregnancy. BJOG 2007;114:253-63.

10. Condous GS. Ultrasound diagnosis of ectopic pregnancy. Semin Reprod Med 2007;25:85-91.

11. Godin PA, Bassil S, Donnez J. An ectopic pregnancy developing in a previous cesarean section scar. Fertil Steril 1997;67:398-400.

12. Lipscomb G. Medical therapy for ectopic pregnancy. Semin Reprod Med 2007;25:93-8.

13. Graesslin O, Dedecker F, Quereux C, Gabriel R. Conservative treatment of ectopic pregnancy in a cesarean scar. Obstet Gynecol 2005;105:869-71.

14. Wang CJ, Chao AS, Yuen LT, Wang CW, Soong YK, Lee CL. Endoscopic management of cesarean scar pregnancy. Fertil Steril 2006;85:494.e1-4.

15. Al-Sunaidi M, Tulandi T. Surgical treatment of ectopic pregnancy. Semin Reprod Med 2007;25:117-22.

16. Mitra AG, Harris-Owens M. Conservative medical management of advanced cervical ectopic pregnancies. Obstet Gynecol Surv 2000;55:385-9. 